\title{
Dark Soliton Interaction of Spinor Bose-Einstein Condensates in an Optical Lattice
}

\author{
Zai-Dong Li, and Qiu-Yan Li \\ Department of Applied Physics, Hebei University of Technology, Tianjin 300130, China
}

We study the magnetic soliton dynamics of spinor Bose-Einstein condensates in an optical lattice which results in an effective Hamiltonian of anisotropic pseudospin chain. An equation of nonlinear Schrödinger type is derived and exact magnetic soliton solutions are obtained analytically by means of Hirota method. Our results show that the critical external field is needed for creating the magnetic soliton in spinor Bose-Einstein condensates. The soliton size, velocity and shape frequency can be controlled in practical experiment by adjusting the magnetic field. Moreover, the elastic collision of two solitons is investigated in detail.

PACS numbers: 03.75.Lm, 05.30.Jp, 67.40.Fd

\section{INTRODUCTION}

The realization of spinor Bose-Einstein condensates (BECs) opens a useful tool to understand and to confirm the dynamics of periodic structures in solid state physics. Recently, the research of spinor BECs trapped in optical potentials have received much attention both experimentally [1, 2] and theoretically [3]. Due to the internal degrees of freedom for the hyperfine spin of the atoms, spinor BECs bring forth a rich variety of phenomena such as spin domains [4, 5] and textures [6]. When the potential valley is so deep that the individual sites are mutually independent, spinor BECs at each lattice site behave like spin magnets and can interact with each other through both the light-induced and the static, magnetic dipole-dipole interactions. These site-to-site dipolar interactions can cause the ferromagnetic phase transition [7, 8] leading to a "macroscopic" magnetization of the condensate array, the spin-wave like excitation [7-10] and magnetic soliton [11, 12] analogous to the spin-wave and magnetic soliton in a ferromagnetic spin chain. For a practical spin chain, the site-to-site interaction is caused mainly by the exchange interaction, while the dipole-dipole interaction is negligibly small. For the spinor BECs in the optical lattice, the exchange interaction is 
absent. The individual spin magnets are coupled by the magnetic and the light-induced dipole-dipole interactions [9, 10] which are no longer negligible due to the large number of atoms $N$ at each lattice site, typically of the order of 1000 or more. Therefore, the spinor BECs in an optical lattice offer a totally new environment to study spin dynamics in periodic structures. The magnetic soliton excited by the interaction between the spin waves is an important and interesting phenomenon in spinor BECs. The Heisenberg model of spin-spin interactions plays a significant role in understanding many complex magnetic structures in solids. It explains the existence of ferromagnetism and antiferromagnetism at temperatures below the Curie temperature. The magnetic soliton [13], which describes localized magnetization, is an important nonlinear excitation in the Heisenberg model [14 17]. However, the generation of controllable solitons is an extremely difficult task due to the complexity of the conventional magnetic materials. The spinor BECs seems an ideal system to serve as a new test ground for studying the nonlinear excitations of spin waves both theoretically and experimentally.

In this paper, we demonstrate that the magnetic soliton and elastic soliton collision are admitted for spinor BECs in a one-dimensional optical lattice. By means of Hirota method we obtain the analytical dark soliton solutions, and also discuss the soliton interaction in detail. The outline of this paper is organized as follows: In Sec. II the Solitons in a Spin Chain of Atomic BEC's is investigated in detail. Next, we obtain the one-soliton solution of spinor BECs in an optical lattice. In Sec. VI, the general two-soliton solution is obtained. Analysis reveals that elastic soliton collision occurs and there is a phase exchange during collision. Finally, our concluding remarks are given in Sec. V.

\section{SOLITONS IN A SPIN CHAIN OF ATOMIC BEC'S}

The Hamiltonian describing an $F=1$ spinor condensate trapped in an optical lattice, which is subject to the magnetic dipole-dipole interactions $H_{d}$ and is coupled to an external magnetic field via the magnetic dipole Hamiltonian $H_{B}$ has the form as [3, 4, 6 , 7 ]

$$
H=H_{0}+H_{d}+H_{B},
$$


where the first term $H_{0}$ describes the interaction of the atoms with the lattice potential $U_{L}(\mathbf{r})$ and the ground-state collisions which can be written as

$$
\begin{aligned}
H_{0}= & \sum_{\alpha} \int d \mathbf{r} \hat{\psi}_{\alpha}^{\dagger}(\mathbf{r})\left[-\frac{\hbar^{2} \nabla^{2}}{2 m}+U_{L}(\mathbf{r})\right] \hat{\psi}_{\alpha}(\mathbf{r}) \\
& +\sum_{\alpha, \beta, \mu, \nu} \frac{\lambda_{s}}{2} \int d \mathbf{r} \hat{\psi}_{\alpha}^{\dagger}(\mathbf{r}) \hat{\psi}_{\beta}^{\dagger}(\mathbf{r}) \hat{\psi}_{\beta}\left(\mathbf{r}^{\prime}\right) \hat{\psi}_{\alpha}(\mathbf{r}) \\
& +\sum_{\alpha, \beta, \mu, \nu} \frac{\lambda_{a}}{2} \int d \mathbf{r} \hat{\psi}_{\alpha}^{\dagger}(\mathbf{r}) \hat{\psi}_{\mu}^{\dagger}(\mathbf{r}) \mathbf{F}_{\alpha \beta} \cdot \mathbf{F}_{\mu \nu} \hat{\psi}_{\nu}(\mathbf{r}) \hat{\psi}_{\beta}(\mathbf{r}),
\end{aligned}
$$

where $\hat{\psi}_{\alpha}(r)$ is the field annihilation operator for an atom in the hyperfine state $\left|f=1, m_{f}=\alpha\right\rangle$, the indices $\alpha, \beta, \mu, \nu=0, \pm 1$ denote the Zeeman sublevels of the ground state, the parameters $\lambda_{s}$ and $\lambda_{a}$ characterize the short-range spin-independent and spinchanging $s$-wave collisions, respectively. When the optical lattice potential is deep enough there is no spatial overlap between the condensates at different lattice sites. Under this condition the tight-binding approximation is reasonable that the atomic field operator can be expanded as $\hat{\psi}(\mathbf{r})=\sum_{n} \sum_{\alpha=0, \pm 1} \hat{a}_{\alpha}(n) \phi_{n}(\mathbf{r})$, where $n$ labels the lattice sites, $\phi_{n}(\mathbf{r})$ is the Hartree wave function of the condensate for the $n$th microtrap and the operators $\hat{a}_{\alpha}(n)$ satisfy the bosonic commutation relations $\left[\hat{a}_{\alpha}(n), \hat{a}_{\beta}^{\dagger}(l)\right]=\delta_{\alpha \beta} \delta_{n l}$. It is assumed that all Zeeman components share the same spatial wave function. If the condensates at each lattice site contain the same number of atoms $N$, the ground-state wave functions for different sites have the same form $\phi_{n}(\mathbf{r})=f\left(\mathbf{r}-\mathbf{r}_{n}\right)$. In this case the dipole-dipole interaction potential $H_{d}$ in Eq. (11) can be expressed by

$$
H_{d}=\sum_{i} \sum_{j(\neq i)} \frac{\mu_{0}}{4 \pi\left|\mathbf{r}_{i j}\right|^{3}}\left[\vec{\mu}_{i} \cdot \vec{\mu}_{j}-3\left(\vec{\mu}_{i} \cdot \hat{\mathbf{r}}_{i j}\right)\left(\vec{\mu}_{j} \cdot \hat{\mathbf{r}}_{i j}\right)\right],
$$

where the parameter $\vec{\mu}_{i}=\gamma \mathbf{S}_{i}$ is the magnetic dipole moment at site $i$, with $\mathbf{S}_{i}=$ $\hat{a}_{\alpha}^{\dagger}(i) \mathbf{F}_{\alpha \beta} \hat{a}_{\beta}(i)$ being the total angular momentum operator and $\gamma$ the gyromagnetic ratio, $\mathbf{r}_{i}$ denotes the coordinate of the $i$ th site, $\mathbf{r}_{i j}=\mathbf{r}_{i}-\mathbf{r}_{j}, \hat{\mathbf{r}}_{i j}=\mathbf{r}_{i j} /\left|\mathbf{r}_{i j}\right|, \mu_{0}$ is the vacuum permeability. Also the Zeeman energy $H_{B}$ can be described by

$$
H_{B}=-\gamma \sum_{i} \mathbf{S}_{i} \cdot \mathbf{B}
$$

where $\mathbf{B}$ is the external magnetic field.

In this paper we consider a one-dimensional optical lattice along the $z$-direction, which is also chosen as the quantization axis. In the absence of spatial overlap between individual 
condensates, and neglecting unimportant constants, the effective spin Hamiltonian can be constructed [7, 9] as

$$
H=\sum_{i}\left[\lambda_{a}^{\prime} \mathbf{S}_{i}^{2}+\sum_{j(\neq i)} J_{i j} \mathbf{S}_{i} \cdot \mathbf{S}_{j}-3 \sum_{j(\neq i)} J_{i j} S_{i}^{z} S_{j}^{z}-\gamma \mathbf{S}_{i} \cdot \mathbf{B}\right]
$$

which determine the dynamics of the magnetic dipole moment of spinor BECs in an optical lattice, where the parameter $\lambda_{a}^{\prime}=(1 / 2) \lambda_{a} \int d^{3} r\left|\phi_{n}(\mathbf{r})\right|^{4}$, here $\lambda_{a}$ characterizes the spindependent $s$-wave collisions, and $J_{i j}=\gamma^{2} \mu_{0} /\left(4 \pi\left|\mathbf{r}_{i j}\right|^{3}\right)$. The first term in Eq. (2) represent the spin-dependent interatomic collisions at a given site, the second and the third terms describe the site-to-site spin coupling induced by the static magnetic field dipole-dipole interaction. Without this interaction terms, the ground state of the Hamiltonian (2) is $|G S\rangle=|N,-N\rangle$ where $N=\sum_{i} N_{i}$ is the total atomic number in the lattice. The total spin at site $i$ has the expectation value $\left\langle\hat{S}_{i}^{z}\right\rangle=-N_{i} \hbar$. Due to the large factor $N_{i}$, the magnetic dipole-dipole interaction in the optical lattice can not be neglected. After the site-to-site coupling is considered, the transfer of the transverse spin excitation from site to site is allowed, resulting in the distortion of the ground state spin structure. This distortion can propagate and hence generate magnetic soliton or spin wave along the atomic spin chain.

It should be noted that the Holstein-Primakoff transformations [18] reported earlier is an useful tool to clear the physical meaning of spin waves and magnetic soliton. For this transformation a local spin-deviation operator is introduced firstly, i.e., $\hat{n}=\hat{S}-\hat{S}^{z}$ with the eigenvalues $n=S-m$ in which it can be seen that the increasing $m$ decreases $n$ and vice versa. For the state $|n\rangle$, the $\hat{S}^{-}|n\rangle=\sqrt{(2 S-n)(1+n)}|n+1\rangle$ and $\hat{S}^{+}|n\rangle=$ $\sqrt{(2 S-n)(n-1)}|n-1\rangle$, it can define the creation and annihilation operators $a$ and $a^{+}$ which satisfy the following boson commutator relation $\left[\hat{a}, \hat{a}^{+}\right]=1,[\hat{a}, \hat{a}]=\left[\hat{a}^{+}, \hat{a}^{+}\right]=0$. The result of $a$ and $a^{+}$operate on the state $|n\rangle$ is $\hat{a}|n\rangle=\sqrt{n}|n-1\rangle, \hat{a}^{+}|n\rangle=\sqrt{n+1}|n+1\rangle$, and $\hat{a}^{+} \hat{a}|n\rangle=n|n\rangle$. With the above relations, the spin operators $\hat{S}$ can be expressed by the Bose operators $\hat{a}^{+}$and $\hat{a}$, i.e., Holstein-Primakoff transformations [18] as follows

$$
\begin{aligned}
& \hat{S}^{+}=\left(\sqrt{2 S-\hat{a}^{+} \hat{a}}\right) \hat{a}, \\
& \hat{S}^{-}=\hat{a}^{+}\left(\sqrt{2 S-\hat{a}^{+} \hat{a}}\right), \\
& \hat{S}_{z}=\left(S-\hat{a}^{+} \hat{a}\right) .
\end{aligned}
$$

In fact the spin waves and magnetic soliton show the small distortion of the ground-state 
spin structure, i.e., $n \ll S$. In this case we can expand the Eq. (3) as

$$
\begin{aligned}
\hat{S}^{+} & =\sqrt{2 S}\left(1-\frac{\hat{a}^{+} \hat{a}}{4 S}+\cdots\right) \hat{a}, \\
\hat{S}^{-} & =\hat{a}^{+} \sqrt{2 S}\left(1-\frac{\hat{a}^{+} \hat{a}}{4 S}+\cdots\right), \\
\hat{S}_{z} & =\left(S-\hat{a}^{+} \hat{a}\right) .
\end{aligned}
$$

Substituting the Eq. (41) into the Hamiltonian (2) and keeping terms through fourth order in $\hat{a}$ and $\hat{a}^{\dagger}$, we can get

$$
\begin{aligned}
H= & N \lambda_{a}^{\prime} S(S+1)-\gamma N S B_{z}+\gamma B_{z} \sum_{i} \hat{a}_{i}^{\dagger} \hat{a}_{i}+S \sum_{i} \sum_{j(\neq i)} J_{i j}\left(\hat{a}_{i} \hat{a}_{j}^{\dagger}+\hat{a}_{i}^{\dagger} \hat{a}_{j}\right) \\
& -\frac{1}{4} \sum_{i} \sum_{j(\neq i)} J_{i j}\left(\hat{a}_{j}^{\dagger} \hat{a}_{j}^{\dagger} \hat{a}_{j} \hat{a}_{i}+\hat{a}_{i}^{\dagger} \hat{a}_{i} \hat{a}_{i} \hat{a}_{j}^{\dagger}+\hat{a}_{i}^{\dagger} \hat{a}_{j}^{\dagger} \hat{a}_{j} \hat{a}_{j}+\hat{a}_{i}^{\dagger} \hat{a}_{i}^{\dagger} \hat{a}_{i} \hat{a}_{j}\right) \\
& -2 \sum_{i} \sum_{j(\neq i)} J_{i j}\left(S^{2}-S\left(\hat{a}_{j}^{\dagger} \hat{a}_{j}+\hat{a}_{i}^{\dagger} \hat{a}_{i}\right)+\hat{a}_{i}^{\dagger} \hat{a}_{i} \hat{a}_{j}^{\dagger} \hat{a}_{j}\right) .
\end{aligned}
$$

Under the spin coherent state and using the time-dependent variation principle, the nonlinear operator motion equation of Hamiltonian (5) can be transformed into an equation for the probability amplitude $\psi_{k}=\left\langle\psi\left|a_{k}\right| \psi\right\rangle$ which describes the nonlinear dynamics of coherent spin excitations on the lattice $k$.

$$
\begin{aligned}
i \hbar \frac{\partial}{\partial t} \psi_{k}= & \left(\gamma B_{z}+4 J S\right) \psi_{k}+2 J S \sum_{\delta} \psi_{k+\delta}-4 J \sum_{\delta} \psi_{k} \psi_{k+\delta}^{*} \psi_{k+\delta} \\
& -\frac{1}{2} J \sum_{\delta}\left(2 \psi_{k}^{*} \psi_{k} \psi_{k+\delta}+\psi_{k+\delta}^{*} \psi_{k+\delta} \psi_{k+\delta}+\psi_{k} \psi_{k} \psi_{k+\delta}^{*}\right)
\end{aligned}
$$

where $\delta= \pm 1$, i.e., we consider only the nearest-neighbor interactions which is a good approximation [19] for the BECs in a one dimensional optical lattice as the large lattice constant.

\section{ONE SOLITON SOLUTION}

When the optical lattice is infinitely long and the spin excitations are in the longwavelength limit, $\psi_{k}, \psi_{k+\delta} \rightarrow \psi(z, t)$ in the continuum limit approximation, we have

$$
-i \frac{\hbar}{J} \psi_{t}+\left(\frac{\gamma B_{z}}{J}+8 S\right) \psi+2 S \psi_{z z}-12 \psi|\psi|^{2}=0 .
$$

In fact, Eq. (7) has dark soliton solutions which can be obtained by many methods. Here we take the Hirota bilinear transformation to get the exact one- and two-soliton solutions 
of Eq. (7). For this method it apply the direct transformation to the nonlinear equation which is the form

$$
\psi=\frac{g}{f},
$$

where $g(z, t)$ are complex functions and $f(z, t)$ is a real function. Substituting (8) into (7) we obtain

$$
-f \frac{\hbar}{J}\left(i D_{t}-\frac{2 J S}{\hbar} D_{z}^{2}\right) g \cdot f-4 S g\left[D_{z}^{2} f \cdot f+\frac{3}{S} g \bar{g}-\left(\frac{\gamma B_{z}}{4 S J}+2\right) f^{2}\right]=0
$$

where $D_{t}$ and $D_{z}^{2}$ are the Hirota bilinear operators which are defined as

$$
D_{z}^{m} D_{t}^{n} g(z, t) \cdot f(z, t)=\left.\left(\frac{\partial}{\partial z}-\frac{\partial}{\partial z^{\prime}}\right)^{m}\left(\frac{\partial}{\partial t}-\frac{\partial}{\partial t^{\prime}}\right)^{n} g(z, t) f\left(z^{\prime}, t^{\prime}\right)\right|_{z=z^{\prime}, t=t^{\prime}} .
$$

Then Eq. (9) can be decoupled as two equations

$$
\begin{aligned}
\hat{G}_{1} g \cdot f & =0, \\
\hat{G}_{2} f \cdot f & =-\frac{3}{S} g \bar{g},
\end{aligned}
$$

where the Hirota bilinear operators $\hat{G}_{1}$ and $\hat{G}_{2}$ are given by

$$
\begin{aligned}
& \hat{G}_{1}=i D_{t}-\frac{2 J S}{\hbar} D_{z}^{2}-\lambda, \\
& \hat{G}_{2}=D_{z}^{2}+\frac{\hbar \lambda}{4 S J}-\frac{\gamma B_{z}}{4 S J}-2,
\end{aligned}
$$

where $\lambda$ is constant to be determined. Now the Eq. (11) has made the Eq. (17) to the normal procedure of Hirota method for getting the exact soliton solutions. In the following by making a series of suitable assumption for the expression of $g$ and $f$, the exact one- and two-soliton solution can be obtained analytically. To this purpose we suppose firstly that

$$
g=g_{0}\left(1+\chi g_{1}\right), f=1+\chi f_{1},
$$

in order to construct a dark one-soliton solution for the system (7), where $\chi$ is an arbitrary parameter which is absorbed in expressing the soliton solutions in the following sections. Substituting (13) into (11), and collecting the coefficients of $\chi^{0}$, we obtain

$$
\begin{aligned}
\hat{G}_{1} g_{0} \cdot 1 & =0 \\
\frac{\hbar \lambda}{4 S J}-\frac{\gamma B_{z}}{4 S J}-2 & =-\frac{3}{S} g_{0} \bar{g}_{0},
\end{aligned}
$$


where the first equation in Eq. (14) can be expressed by the definition (10) from which in is easy to find the solution satisfying (14) as

$$
g_{0}=\tau_{1} \exp \left(i \Phi_{1}\right)
$$

where $\Phi_{1}$ has of form

$$
\Phi_{1}=\sqrt{\frac{\hbar}{2 J S}} l_{1} z-\left(\lambda-l_{1}^{2}\right) t-\Phi_{1}^{0},
$$

in which $l_{1}, \Phi_{1}^{0}$ are real constants and $\tau_{1}$ are complex constant. With the restriction, i.e., the second equation in Eq. (14) the expression of $\tau_{1}$ is

$$
\left|\tau_{1}\right|^{2}=\frac{1}{12 J}\left(\gamma B_{z}+8 S J-\hbar \lambda\right)
$$

which imply the condition $\gamma B_{z}+8 S J-\hbar \lambda>0$, i.e., the minimum external field value for the existence of soliton solution in Eq. (17), $B_{z}=(\hbar \lambda-8 S J) / \gamma$. The coefficient of $\chi$ in Eq. (11) leads to

$$
\begin{aligned}
& £_{1}\left(1 \cdot f_{1}+g_{1} \cdot 1\right)=0, \\
& \hat{G}_{2}\left(f_{1} \cdot 1+1 \cdot f_{1}\right)=-\frac{3}{S}\left|\tau_{1}\right|^{2}\left(g_{1}+\bar{g}_{1}\right),
\end{aligned}
$$

where $£_{1}$ is defined as

$$
£_{1}=i D_{t}-\frac{2 J S}{\hbar} D_{z}^{2}-i 2 l_{1} \sqrt{\frac{2 J S}{\hbar}} D_{z}
$$

By expanding Eq. (18) with the definition (10) in detail, one can easily check that equations (18) admit the following solutions

$$
\begin{aligned}
& g_{1}=Z_{g} \exp \xi_{1}, \\
& f_{1}=\exp \xi_{1},
\end{aligned}
$$

where

$$
\xi_{1}=P_{1} z-\Omega_{1} t-\xi_{1}^{0},
$$

in which $P_{1}, \Omega_{1}$ and $\xi_{1}^{0}$ are real constants and $Z_{g}$ is a complex constant which is given by

$$
Z_{g}=-\frac{\left[\frac{2 J S}{\hbar} P_{1}^{2}-i\left(2 P_{1} l_{1} \sqrt{\frac{2 J S}{\hbar}}+\Omega_{1}\right)\right]^{2}}{\left(\frac{2 J S}{\hbar} P_{1}^{2}\right)^{2}+\left(2 P_{1} l_{1} \sqrt{\frac{2 J S}{\hbar}}+\Omega_{1}\right)^{2}}
$$


From this equation it can easily be seen that $\left|Z_{g}\right|^{2}=1$. Then using Eq. (13), after absorbing $\chi$, the dark one-soliton solution of spinor BECs in an optical lattice can be derived as

$$
\psi_{1}=\frac{g}{f}=\frac{\tau_{1}}{2}\left[\left(1+Z_{g}\right)-\left(1-Z_{g}\right) \tanh \frac{\xi_{1}}{2}\right] \exp \left(i \Phi_{1}\right) .
$$

The solution in Eq. (22) describes a magnetic dark soliton solution characterized by four real parameters: velocity of envelope motion $\Omega_{1} / P_{1}$, phase $\Phi_{1}$, coordinate of the center of the solitary wave $\xi_{1}^{0} / P_{1}$ and initial phase $\sqrt{\frac{2 J S}{\hbar}} \Phi_{1}^{0} / l_{1}$. This solution is similar to that of the Heisenberg spin chain with an external field where the dipolar coupling is typically several orders of magnitude weaker than the exchange coupling and would correspond to Curie temperatures much below the observed ones. Hence its contribution to the spin wave and magnetic soliton can be neglected in practice. However, for the spinor BECs in the optical lattice the exchange interaction is absent and the individual spin magnets are coupled by the magnetic dipole-dipole interactions. Due to the large number of atoms $N$ at each lattice site, these site to site interactions, despite the large distance between sites, explain the natural existence of magnetic soliton which agrees with the results in Refs. [7, 9]. With the help of Eq. (18) we have

$$
\frac{S}{6 P_{1}^{2}}=\frac{\left|\tau_{1}\right|^{2}}{P_{1}^{4}+\left(2 P_{1} l_{1} \sqrt{\frac{\hbar}{2 J S}}+\frac{\hbar}{2 J S} \Omega_{1}\right)^{2}} .
$$

By combine the above presentation with the Eq. (17) we obtained the following relation for $P_{1} \neq 0$

$$
B_{z}=\frac{1}{\gamma}\left[2 J S P_{1}^{2}+\left(2 l_{1} \sqrt{\hbar}+\frac{\hbar}{\sqrt{2 J S}} \frac{\Omega_{1}}{P_{1}}\right)^{2}-8 S J+\hbar \lambda\right],
$$

which shows that the external field $B_{z}$ and the dipole-dipole interaction can affect the velocities, size and shape frequency of the magnetic soliton. It offer an useful scheme to control soliton in practical experiment by adjusting of the magnetic field.

\section{DARK SOLITON INTERACTION}

In this section we will give the analytical expression of two soliton solutions of Eq. (7). The properties is discussed in detail as well. To this purpose for constructing the dark two-soliton solutions of Eq. (7) we now assume that

$$
g=g_{0}\left(1+\chi g_{1}+\chi^{2} g_{2}\right), f=1+\chi f_{1}+\chi^{2} f_{2},
$$


where $g_{0}$ and $h_{0}$ are obtained here as in Eq. (15). By employing the same procedure before we obtain the following set of equations from Eq. (11), corresponding to the different powers of $\chi$ as follows

(i) for the coefficient of $\chi$

$$
\begin{aligned}
& £_{1}\left(1 \cdot f_{1}+g_{1} \cdot 1\right)=0, \\
& \hat{G}_{2}\left(f_{1} \cdot 1+1 \cdot f_{1}\right)=-\frac{3}{S}\left|\tau_{1}\right|^{2}\left(g_{1}+\bar{g}_{1}\right),
\end{aligned}
$$

where $£_{1}$ is same with Eq. (19).

(ii) for the coefficient of $\chi^{2}$

$$
\begin{gathered}
\hat{G}_{1}\left(g_{0} \cdot f_{2}+g_{0} g_{1} \cdot f_{1}+g_{0} g_{2} \cdot 1\right)=0, \\
£_{1}\left(1 \cdot f_{2}+g_{1} \cdot f_{1}+g_{2} \cdot 1\right)=0, \\
\hat{G}_{2}\left(1 \cdot f_{2}+f_{2} \cdot 1+f_{1} \cdot f_{1}\right)=-\frac{3}{S}\left[\left|\tau_{1}\right|^{2}\left(g_{1} \bar{g}_{1}+g_{2}+\bar{g}_{2}\right)\right] .
\end{gathered}
$$

(iii) for the coefficient of $\chi^{3}$

$$
\begin{gathered}
\hat{G}_{1}\left(g_{0} g_{1} \cdot f_{2}+g_{0} g_{2} \cdot f_{1}\right)=0, \\
£_{1}\left(g_{1} \cdot f_{2}+g_{2} \cdot f_{1}\right)=0, \\
\hat{G}_{2}\left(f_{1} \cdot f_{2}+f_{2} \cdot f_{1}\right)=-\frac{3}{S}\left|\tau_{1}\right|^{2}\left(g_{1} \bar{g}_{2}+g_{2} \bar{g}_{1}\right) .
\end{gathered}
$$

(iii) for the coefficient of $\chi^{4}$

$$
\begin{gathered}
\hat{G}_{1}\left(g_{0} g_{2} \cdot f_{2}\right)=0, \\
£_{1}\left(g_{2} \cdot f_{2}\right)=0, \\
\hat{G}_{2}\left(f_{2} \cdot f_{2}\right)=-\frac{3}{S}\left|\tau_{1}\right|^{2} g_{2} \bar{g}_{2} .
\end{gathered}
$$

Repeating the same normal procedure in obtaining the one-soliton solution one can easily get the solutions of the above set of equations as

$$
\begin{aligned}
& g_{1}=Z_{1} \exp \xi_{1}+Z_{2} \exp \xi_{2}, \\
& g_{2}=A_{12} Z_{1} Z_{2} \exp \left(\xi_{1}+\xi_{2}\right), \\
& f_{1}=\exp \xi_{1}+\exp \xi_{2}, \\
& f_{2}=A_{12} \exp \left(\xi_{1}+\xi_{2}\right),
\end{aligned}
$$


where

$$
\begin{gathered}
\xi_{j}=P_{j} z-\Omega_{j} t+\xi_{j}^{0}, \\
Z_{j}=-\frac{P_{j}-i \sqrt{6\left|\tau_{1}\right|^{2} / S-P_{j}^{2}}}{P_{j}+i \sqrt{6\left|\tau_{1}\right|^{2} / S-P_{j}^{2}}}
\end{gathered}
$$

in which $j=1,2$. Noting that

$$
\kappa_{j}=\left(\frac{6\left|\tau_{1}\right|^{2}}{S}-P_{1}^{2}\right)^{1 / 2} \frac{2 J S}{\hbar}
$$

by which we can rewrite $Z_{j}$ and $\Omega_{j}, j=1,2$, as

$$
\begin{aligned}
Z_{j} & =-\frac{\frac{2 J S}{\hbar} P_{j}-i \kappa_{j}}{\frac{2 J S}{\hbar} P_{j}+i \kappa_{j}} \\
\Omega_{j} & =\left(\kappa_{j}-2 l_{1} \sqrt{\frac{2 J S}{\hbar}}\right) P_{j},
\end{aligned}
$$

So from Eq. (25) to Eq. (28) the expression of $A_{12}$ is obtained in the form

$$
A_{12}=\frac{\left(P_{1}-P_{2}\right)^{2}\left(\frac{2 J S}{\hbar}\right)^{2}+\left(\kappa_{1}-\kappa_{2}\right)^{2}}{\left(P_{1}+P_{2}\right)^{2}\left(\frac{2 J S}{\hbar}\right)^{2}+\left(\kappa_{1}-\kappa_{2}\right)^{2}}
$$

Now using Eq. (8), (24) and (29), the dark two-solitons can be found explicitly as follows

$$
\psi_{\text {two }}=\frac{g}{f}=\tau_{1} \exp \left(i \Phi_{1}\right) \frac{1+Z_{1} \exp \xi_{1}+Z_{2} \exp \xi_{2}+A_{12} Z_{1} Z_{2} \exp \left(\xi_{1}+\xi_{2}\right)}{1+\exp \xi_{1}+\exp \xi_{2}+A_{12} \exp \left(\xi_{1}+\xi_{2}\right)}
$$

where all the parameters have been obtained in the previous section. The solution (30) describes a general elastic scattering process of two solitary waves with different center velocities $\Omega_{1} / P_{1}$ and $\Omega_{2} / P_{2}$ and the same phase $\Phi_{1}$. Before collision, they move towards each other, one with velocity $\Omega_{1} / P_{1}$ and the other with $\Omega_{2} / P_{2}$. In order to understand the nature of two-soliton interaction, we analyze the asymptotic behavior of two-soliton solution (30). Asymptotically, the two-soliton waves (30) can be written as a combination of two one-soliton waves (22). The asymptotic form of two-soliton solution in limits $t \rightarrow-\infty$ and $t \rightarrow \infty$ is similar to that of the one-soliton solution (22).

(i) Before collision (limit $t \rightarrow-\infty$ ).

(a) Soliton $1\left(\xi_{1} \approx 0, \xi_{2} \rightarrow-\infty\right)$.

$$
\psi_{t w o} \rightarrow \tau_{1} \exp \left(i \Phi_{1}\right)\left[\left(1+Z_{1}\right)-\left(1-Z_{1}\right) \tanh \frac{\xi_{1}}{2}\right],
$$


(b) Soliton $2\left(\xi_{2} \approx 0, \xi_{1} \rightarrow \infty\right)$.

$$
\psi_{\text {two }} \rightarrow \tau_{1} Z_{1} \exp \left(i \Phi_{1}\right)\left[\left(1+Z_{2}\right)-\left(1-Z_{2}\right) \tanh \frac{\xi_{2}+\delta_{0}}{2}\right],
$$

where the initial coordinate of the center of the solitary wave is removed by $\delta_{0}=\ln A_{12}$.

(ii) After collision (limit $t \rightarrow \infty$ ).

(a) Soliton $1\left(\xi_{1} \approx 0, \xi_{2} \rightarrow \infty\right)$.

$$
\psi_{t w o} \rightarrow Z_{2} \tau_{1} \exp \left(i \Phi_{1}\right)\left[\left(1+Z_{1}\right)-\left(1-Z_{1}\right) \tanh \frac{\xi_{1}+\delta_{0}}{2}\right],
$$

(b) Soliton $2\left(\xi_{2} \approx 0, \xi_{1} \rightarrow-\infty\right)$.

$$
\psi_{\text {two }} \rightarrow \tau_{1} \exp \left(i \Phi_{1}\right)\left[\left(1+Z_{2}\right)-\left(1-Z_{2}\right) \tanh \frac{\xi_{1}}{2}\right],
$$

Analysis reveals that there is no amplitude exchange for soliton 1 and soliton 2 during collision because of $\left|Z_{j}\right|=1, j=1,2$. However, from Eqs. (31) to (34) one can see that there is a phase exchange $\delta_{0} / 2$ for soliton 1 and soliton 2 during collision. It shows that the information held in each soliton will almost not be disturbed by each other in soliton propagation. These properties may have potential application in future quantum communication.

\section{CONCLUSION}

Dark soliton dynamics of spinor BECs in an optical lattice is studied in terms of a nonlinear schrödinger equation by means of Hirota method. Exact soliton solutions are obtained analytically and the elastic collision of two solitons is demonstrated. It should be interesting to discuss how to create and to detect such magnetic soliton in experiment. Using Landau-Zener rf-sweeps at high fields (30 G) [4] a condensate was prepared in the hyperfine state $\left|f=1, m_{f}=0\right\rangle$ of sodium, i.e. the ground state of the spinor BECs. Then the atoms of the ground state can be excited to the hyperfine state $\left|f=1, m_{f}= \pm 1\right\rangle$ by laser light experimentally. Therefore the excited state of the spinor BECs, i.e. the magnetic soliton can be created. As the same discussion before [12] the spatial-temporal spin variations in the soliton state are significant. This makes it possible to make a direct detection of the magnetic soliton of spinor BECs. By counting the difference numbers of the population between the spin +1 and -1 Zeeman sublevel, the average of spin component $\left\langle S^{z}\right\rangle$ is 
measured directly. Transverse components can be measured by use of a short magnetic pulse to rotate the transverse spin component to the longitudinal direction. Any optical or magnetic method which can excite the internal transitions between the atomic Zeeman sublevels can be used for this purpose. In current experiments in optical lattices, the lattice number is in the range of 10-100, and each lattice site can accommodate a few thousand atoms. This leads to a requirement for the frequency measurement precision of about 10-100 $\mathrm{kHz}$. This is achievable with current techniques.

The magnetic soliton of spinor BECs in an optical lattice is mainly caused by the magnetic and the light-induced dipole-dipole interactions between different lattice sites. Since these long-range interactions are highly controllable the spinor BECs in optical lattice which is an exceedingly clean system can serve as a test ground to study the static and dynamic aspects of soliton excitations.

\section{ACKNOWLEDGEMENT}

This work is supported by the Nature Science Foundation of China No. 10647122, the Doctoral Foundation of Education Bureau of Hebei Province of China No. 2006110 and the key subject construction project of Heibei Provincial University of China.

[1] J. Stenger, S. Inouye, D. M. Stamper-Kurn, H. J. Miesner, A. P. Chikkatur and W. Ketterle, Nature (London) 396 (1998) 345.

[2] B. P. Anderson and M. A. Kasevich, Science 282 (1998) 1686;

D. M. Stamper-Kurn, M. R. Andrews, A. P. Chikkatur, S. Inouye, H.-J. Miesner, J. Stenger, and W. Ketterle, Phys. Rev. Lett. 80 (1998) 2027.

[3] T. L. Ho, Phys. Rev. Lett. 81 (1998) 742;

C. K. Law, T. Ohmi and K. Machida, J. Phys. Soc. Jpn. 67 (1998) 1822;

C. K. Law, H. Pu and N. P. Bigelow, Phys. Rev. Lett. 81 (1998) 5257;

Y. Wu and X. Yang, J. Opt. Soc. Am. B 23 (2006) 1888; Optics Letters 30, No. 3 (2005) 311; Phys. Rev. A. 68 (2003) 013608.

[4] H. J. Miesner, D. M. Stamper-Kurn, J. Stenger, S. Inouye, A. P. Chikkatur and W. Ketterle, 
Phys. Rev. Lett. 82 (1999) 2228;

Jun'ichi Ieda, Takahiko Miyakawa, and Miki Wadati, Phys. Rev. Lett. 93 (2004) 194102.

[5] N. Gemelke, E. Sarajlic, Y. Bidel, S. Hong, and S. Chu, Phys. Rev. Lett. 95 (2005) 170404.

Wenxian Zhang, D. L. Zhou, M.-S. Chang, M. S. Chapman, and L. You, Phys. Rev. Lett. 95 (2005) 180403.

[6] T. Ohmi and K. Machida, J. Phys. Soc. Jpn. 67 (1999) 1822.

[7] H. Pu, W. P. Zhang and P. Meystre, Phys. Rev. Lett. 87 (2001) 140405.

[8] K. Gross, C. P. Search, H. Pu, W. P. Zhang, and P. Meystre, Phys. Rev. A. 66 (2002) 033603.

[9] W. P. Zhang, H. Pu, C. Search, and P. Meystre, Phys. Rev. Lett. 88 (2002) 060401;

K. Góral, L. Santos, and M. Lewenstein, Phys. Rev. Lett. 88 (2002) 170406.

[10] M. Rigol, V. Rousseau, R. T. Scalettar, and R. R. P. Singh, Phys. Rev. Lett. 95 (2005) 110402; J. N. Fuchs, D. M. Gangardt, T. Keilmann, and G. V. Shlyapnikov, Phys. Rev. Lett. 95 (2005) 150402 .

[11] Z. -W. Xie, W. Zhang, S. T. Chui, and W. M. Liu, Phys. Rev. A 69 (2004) 053609;

Z. -W. Xie, Ze-Xian Cao, E. I. Kats, and W. M. Liu, Phys. Rev. A 71 (2005) 025601.

[12] Zai-Dong Li, P. B. He, Lu Li, J.-Q. Liang, and W. M. Liu, Phys. Rev. A 71053611 (2005);

Lu Li, Zaidong Li, Boris A. Malomed, Dumitru Mihalache, and W. M. Liu, Phys. Rev. A 72 (2005) 033611.

[13] A. M. Kosevich, B. A. Ivanoy and A. S. Kovalev, Phys. Rep. 194 (1990) 117.

[14] J. Tjon and J. Wright, Phys. Rev. B 15 (1977) 3470.

[15] Z. D. Li, L. Li, W. M. Liu, J. Q. Liang and T. Ziman, Phys. Rev. E 68 (2003) 036102;

Z. D. Li, J. Q. Liang, L. Li, W. M. Liu, Phys. Rev. E 69 (2004) 066611;

Z. D. Li, L. Li, J. Q. Liang, Chin. Phys. Lett 20 (2003) 39;

Chin. Phys. Lett 21 (2004) 443;

Q. Y. Li, Z. W. Xie, L. Li, Z. D. Li, and J. Q. Liang, Annals of Physics 312 (2004) 128.

[16] M. J. Ablowitz and P. A. Clarkson, Solitons, Nonlinear Evolution Equations and Inverse Scattering (Cambridge University Press, New York, 1991).

[17] N. N. Huang, Z. Y. Chen and Z. Z. Liu, Phys. Rev. Lett. 75 (1995) 1395.

[18] T. Holstein, H. Primakoff, Phys. Rev. 58 (1940) 1098.

[19] V. V. Konotop, M. Salerno, and S. Takeno, Phys. Rev. E 56 (1997) 7240. 